\title{
The prognostic value of platelet-to-lymphocyte ratio on the long-term renal survival in patients with IgA nephropathy
}

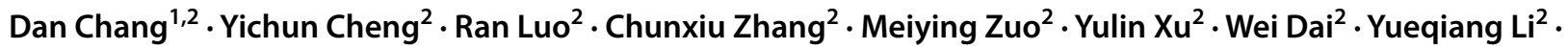 \\ Min $\mathrm{Han}^{2} \cdot$ Xiaofeng $\mathrm{He}^{2}$. Shuwang $\mathrm{Ge}^{2}\left[\right.$ (]) Gang $\mathrm{Xu}^{2}$
}

Received: 10 April 2020 / Accepted: 14 September 2020 / Published online: 28 October 2020

(c) The Author(s) 2020

\begin{abstract}
Purpose Platelet-to-lymphocyte ratio (PLR) was established showing the poor prognosis in several diseases, such as malignancies and cardiovascular diseases. But limited study has been conducted about the prognostic value of PLR on the longterm renal survival of patients with Immunoglobulin A nephropathy (IgAN).

Methods We performed an observational cohort study enrolling patients with biopsy-proven IgAN recorded from November 2011 to March 2016. The definition of composite endpoint was eGFR decrease by $50 \%$, eGFR $<15 \mathrm{~mL} / \mathrm{min} / 1.73 \mathrm{~m}^{2}$, initiation of dialysis, or renal transplantation. Patients were categorized by the magnitude of PLR tertiles into three groups. The Kaplan-Meier curves and multivariate Cox models were performed to determine the association of PLR with the renal survival of IgAN patients.

Results 330 patients with a median age of 34.0 years were followed for a median of 47.4 months, and 27 patients $(8.2 \%)$ had reached the composite endpoints. There were no differences among the three groups (PLR $<106,106 \leq$ PLR $\leq 137$, and PLR > 137) in demographic characteristics, mean arterial pressure (MAP), proteinuria, and estimated glomerular filtration rate (eGFR) at baseline. The Kaplan-Meier curves showed that the PLR $>137$ group was significantly more likely to poor renal outcomes than the other two groups. Using univariate and multivariate cox regression analyses, we found that PLR $>137$ was an independent prognostic factor for poor renal survival in patients with IgAN. Subgroup analysis revealed that the PLR remained the prognostic value for female patients or patients with eGFR less than $60 \mathrm{~mL} / \mathrm{min} / 1.73 \mathrm{~m}^{2}$.

Conclusions Our results underscored that baseline PLR was an independent prognostic factor for poor renal survival in patients with IgAN, especially for female patients or those patients with baseline eGFR less than $60 \mathrm{~mL} / \mathrm{min} / 1.73 \mathrm{~m}^{2}$.
\end{abstract}

Keywords Immunoglobulin A nephropathy $\cdot$ Platelet-to-lymphocyte ratio $\cdot$ Renal survival $\cdot$ Cohort

\section{Abbreviations}

PLR Platelet-to-lymphocyte ratio

IgAN Immunoglobulin A nephropathy

ESRD End-stage renal disease

Dan Chang and Yichun Cheng contributed equally to this work.

Shuwang Ge

geshuwang@tjh.tjmu.edu.cn

$\triangle$ Gang Xu

xugang@tjh.tjmu.edu.cn

1 Department of Nephrology, University of Electronic Science and Technology, Sichuan Academy of Sciences and Sichuan Provincial People's Hospital, Chengdu, Sichuan, China

2 Department of Nephrology, Tongji Hospital of Tongji Medical College of Huazhong University of Science and Technology, Wuhan, Hubei, China
SBP Systolic blood pressure

DBP Diastolic blood pressure

MAP Mean arterial pressure

WBC White blood cell count

Scr Serum creatinine

UA Uric acid

eGFR Estimated glomerular filtration rate

MDRD Modification of Diet in Renal Disease Study

SD Standard deviation

IQR Interquartile range

HRs Hazard ratios

CIs Confidence intervals

RAAS Rein-angiotensin-aldosterone-system

IS Immune suppression 


\section{Introduction}

Immunoglobulin A nephropathy ( $\operatorname{IgAN}$ ) is one of the most common primary glomerulonephritis worldwide [1]. The number of patients with IgAN accounts for $58.2 \%$ of glomerulonephritis in China [2]. Studies have shown that $15 \%$ to $40 \%$ of patients with IgAN develop the end-stage renal disease (ESRD) by 10 to 20 years after diagnosis $[3,4]$. As one of the main reasons to increase the social burden, however, it is still challenging to precisely predict the outcomes of IgAN patients [5,6]. Several clinical indicators such as renal function and proteinuria at biopsy are demonstrated to be associated with the renal outcome of IgAN [7-9]. But we need to find new risk factors of IgAN to timely prevent the disease from deterioration.

Platelet-to-lymphocyte ratio (PLR), calculated as platelet count divided by the lymphocyte count, is an inexpensive, replicable, and easily measurable index. There is increasing evidence suggesting that PLR may serve as a novel inflammatory marker and prognostic factors for various diseases, such as malignancies [10-13], cardiovascular diseases [14, 15], chronic obstructive pulmonary disease [16] and saphenous vein graft disease [17].

Although the pathogenesis of IgAN is not completely understood, multiple mechanisms may be involved, and inflammation is known to play a key role [18-21]. However, the clinical significance of PLR in the IgAN process remains unclear. Hence, we conducted this study to explore the prognostic value of PLR on long-term renal survival among patients with IgAN.

\section{Materials and methods}

\section{Study design and study population}

Three hundred and thirty patients with IgAN were recruited in the Tongji hospital affiliated to Tongji medical college of Huazhong University of Science and Technology from November 1, 2011, to March 1, 2016 (Fig. 1). We included biopsy-proven IgAN patients with complete clinical and pathological data. And we excluded patients with blood system disease, connective tissue disease, active infection, and baseline eGFR less than $30 \mathrm{~mL} / \mathrm{min} / 1.73 \mathrm{~m}^{2}$. Patients who received immunosuppressant, glucocorticoid, or nonsteroidal anti-inflammatory drugs treatment at the time of renal biopsy were also excluded.

\section{Demographic and clinical data}

Baseline demographic, clinical and laboratory data were collected from all patients at the time of renal biopsy, including age, gender, systolic blood pressure (SBP), diastolic blood pressure (DBP), white blood cell count (WBC), platelet count, lymphocyte count, hemoglobin, serum albumin, serum creatinine ( $\mathrm{Scr}$ ), uric acid (UA), estimated glomerular filtration rate (eGFR), and proteinuria quantity.

PLR was calculated as the ratio of the absolute platelet count to the absolute lymphocyte count on preoperative routine blood tests.

The blood pressure of patients was measured by a cuff pressure method using a mercury sphygmomanometer or an electronic sphygmomanometer. Mean arterial pressure (MAP) was calculated by $(\mathrm{SBP}+2 \times \mathrm{DBP}) / 3$.

eGFR is calculated by the Modification of Diet in Renal Disease Study (MDRD) equation:
Fig. 1 Flow chart of the patients included in the study

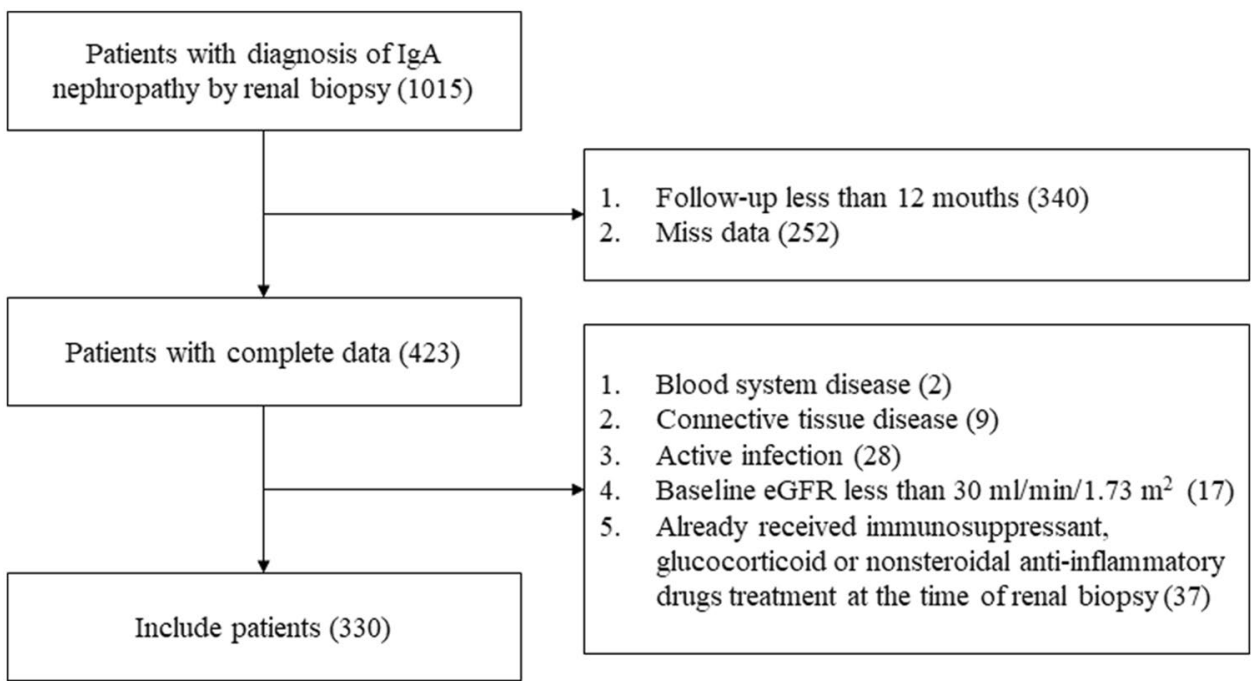


$186 \times(\text { Creatinine } / 88.4)^{-1.154} \times(\text { Age })^{-0.203} \times(0.742$ if female $)$ $\times(1.210$ if black $)$ [22].

Regular visits at intervals of 6 months were performed in every patient. Urine sediment and renal function were tested at every visit throughout follow-up. All follow-up data were updated to March 1, 2019.

\section{Study outcomes}

For survival analysis, the definition of composite endpoint was eGFR decrease by $50 \%$ or ESRD. ESRD was defined as eGFR $<15 \mathrm{~mL} / \mathrm{min} / 1.73 \mathrm{~m}^{2}$, initiation of dialysis, or renal transplantation. Patients were censored at the time of endpoint or loss of follow-up.

\section{Statistical analysis}

The distributions of quantitative variables were assessed for normality. Continuous variables were expressed as the mean \pm standard deviation (SD) (normally distributed variables) or median and interquartile range (IQR) (non-normally distributed variables). For continuous data, one-way analysis of variance was used if the variable was normally distributed; if not, Kruskal-Wallis tests were performed. Categorical data were expressed as frequencies and percentages (\%) and compared by Pearson chi-squared tests or Fisher's exact test, as appropriate. The probabilities of cumulative renal survival curves were generated by the Kaplan-Meier method, and the differences between curves were analyzed by a log-rank test. Univariate and multivariate Cox regression proportional hazards models were built to evaluate independent risk factors of renal progression. No violations of the Cox proportional hazards assumptions were detected. The results of Cox regression analyses were expressed as hazard ratios (HRs) with $95 \%$ confidence intervals (CIs). $P$ values $\leq 0.05$ were considered to indicate statistical significance with 95\% CIs. Statistical Package for the Social Sciences (version 22.0, 2013, IBM SPSS Statistics for Windows, Armonk, NY: IBM Corp) and R software (version 3.5.1, 2013, R Foundation for Statistical Computing, Vienna, Austria. URL https://www.R-project.org/) were used for all statistical analyses.

\section{Results}

The main characteristics of the included patients are shown in Table 1. A total of 330 cases were enrolled in this study, including 137 (41.5\%) men and 193 (58.5\%) women with a median age of 34.0 years and a median PLR of 120.4. Patients were followed for a median of 47.4 months.

Patients were categorized by the magnitude of PLR tertiles into three groups: group 1 with PLR $<106(n=111)$, group 2 with $106 \leq \operatorname{PLR} \leq 137(n=109)$ while group 3 with PLR $>137(n=110)$ (Table 1). There were no significant differences among the three groups in demographic characteristics, MAP, proteinuria, and renal function at baseline. And No significant differences were found among the three groups in the use of rein-angiotensin-aldosterone-system (RAAS) blockade and immune suppression (IS) treatments. Patients with higher PLR value had higher platelet count and lower lymphocyte count. There were $2(0.6 \%)$ patients with a low level of platelet $\left(<100 \times 10^{9} / \mathrm{L}\right)$ and $30(9.1 \%)$ patients with a high level of platelet $\left(>300 \times 10^{9} / \mathrm{L}\right)$. At the end of follow-up, 27 patients $(8.2 \%)$ had reached the composite endpoints, including 17 patients with eGFR decrease by $50 \%$ and 10 patients with ESRD.

The Kaplan-Meier curve showed the association of PLR with poor renal outcomes among IgAN patients (Fig. 2). As no significant difference in the Kaplan-Meier curve was seen between the first and the second tertile of the PLR group, we put the two groups together as PLR $\leq 137$ group. Univariate analysis by Cox regression revealed that hemoglobin, serum albumin, Scr, UA, eGFR, and proteinuria at the time of renal biopsy and use of IS treatments were factors significantly associated with renal survival, which was defined by a status free of composite endpoints (Table 2). Besides, the lymphocyte count and platelet count were not associated with poor renal outcomes. When compared with group PLR $\leq 137$, PLR $>137$ was associated with a higher risk (HR 3.10; 95\% CI 1.44-6.64; $P=0.004$ ) of experiencing the poor renal outcomes. Moreover, after adjusted for age, sex, hemoglobin, serum albumin, Scr, UA, eGFR, proteinuria, and use of IS treatments, PLR $>137$ (HR 2.79; 95\% CI $1.08-7.26 ; P=0.035$ ) was still associated with poor renal outcomes among IgAN patients compared with PLR $\leq 137$ (Table 3).

To be more precise, we also analyzed the renal survival of patients according to the sex, baseline eGFR, and proteinuria categories. As shown in Fig. 3, among female patients, PLR $>137$ was associated with poor renal outcomes compared with PLR $\leq 137$ after adjusted for age, hemoglobin, serum albumin, Scr, UA, eGFR, proteinuria, and use of IS treatments. We also found that patients with baseline eGFR lower than $60 \mathrm{~mL} / \mathrm{min} / 1.73 \mathrm{~m}^{2}$ had a significantly better renal survival in patients with PLR $\leq 137$ than PLR $>137$. While no significant difference was observed between PLR $\leq 137$ and PLR $>137$ groups in male patients or patients whose baseline eGFR higher than $60 \mathrm{~mL} / \mathrm{min} / 1.73$ $\mathrm{m}^{2}$, baseline proteinuria higher than $1 \mathrm{~g} / 24 \mathrm{~h}$ or lower than $1 \mathrm{~g} / 24 \mathrm{~h}$. 
Table 1 Clinical characteristics at the time of renal biopsy and outcomes of included patients categorized by the magnitude of PLR tertiles

\begin{tabular}{|c|c|c|c|c|c|c|}
\hline Variables & All & PLR $<106$ & $106 \leq \mathrm{PLR} \leq 137$ & PLR > 137 & $P$-value & Test statistic \\
\hline \multicolumn{7}{|l|}{ At the time of renal biopsy } \\
\hline Number of patients $(n)$ & 330 & 111 & 109 & 110 & & \\
\hline Age (years, median [IQR]) & $34.0[15]$ & $36.0[16]$ & $34.0[15]$ & $33.0[16]$ & 0.598 & $1.030^{\mathrm{a}}$ \\
\hline Male $(n, \%)$ & $137(41.5)$ & $49(44.1)$ & $44(40.4)$ & $44(40.0)$ & 0.787 & $0.479^{\mathrm{b}}$ \\
\hline MAP (mmHg, mean (SD)) & $97.8(13.7)$ & $96.6(14.1)$ & $98.3(13.1)$ & $98.5(13.9)$ & 0.542 & $0.613^{\mathrm{c}}$ \\
\hline $\mathrm{WBC}\left(\times 10^{9} / \mathrm{L}\right.$, median $\left.[\mathrm{IQR}]\right)$ & $6.79[2.39]$ & $7.06[2.57]$ & $6.79[2.28]$ & $6.34[2.51]$ & 0.163 & $3.624^{\mathrm{a}}$ \\
\hline Lymphocytes $\left(\times 10^{9} / \mathrm{L}\right.$, median $\left.[\mathrm{IQR}]\right)$ & $1.84[0.75]$ & $2.27[0.70]$ & $1.88[0.55]$ & $1.54[0.51]$ & $<0.001$ & $89.433^{\mathrm{a}}$ \\
\hline Platelets $\left(\times 10^{9} / \mathrm{L}\right.$, median $\left.[\mathrm{IQR}]\right)$ & $222.0[83.5]$ & $187.0[77.0]$ & $225.0[73.0]$ & $252.0[77.2]$ & $<0.001$ & $60.560^{\mathrm{a}}$ \\
\hline Hemoglobin (g/L, mean (SD)) & $130.9(20.2)$ & $132.2(18.2)$ & $131.7(21.7)$ & $128.8(20.5)$ & 0.392 & $0.940^{\mathrm{c}}$ \\
\hline Serum albumin $(\mathrm{g} / \mathrm{l}$, mean $(\mathrm{SD}))$ & $39.3(5.7)$ & $39.8(4.5)$ & $39.3(5.7)$ & $38.9(6.7)$ & 0.440 & $0.869^{\mathrm{c}}$ \\
\hline $\operatorname{Scr}(\mu \mathrm{mol} / \mathrm{L}$, median $[\mathrm{IQR}])$ & $83.5[42.0]$ & $83.0[41.0]$ & $86.0[42.5]$ & $82.0[47.3]$ & 0.799 & $0.449^{\mathrm{a}}$ \\
\hline $\mathrm{UA}(\mu \mathrm{mol} / \mathrm{L}$, mean $(\mathrm{SD}))$ & $356.2(99.2)$ & $359.2(90.4)$ & $352.0(94.6)$ & $357.3(112.2)$ & 0.858 & $0.172^{\mathrm{c}}$ \\
\hline eGFR $\left(\mathrm{ml} / \mathrm{min} / 1.73 \mathrm{~m}^{2}\right.$, median $\left.[\mathrm{IQR}]\right)$ & $89.5[47.5]$ & $92.6[45.2]$ & $83.7[45.7]$ & $88.9[55.6]$ & 0.778 & $0.503^{\mathrm{a}}$ \\
\hline Proteinuria (g/24 h, median [IQR]) & $0.78[1.34]$ & $0.96[1.00]$ & $1.03[1.52]$ & $0.63[1.57]$ & 0.209 & $3.135^{\mathrm{a}}$ \\
\hline \multicolumn{7}{|l|}{ Follow-up and treatment } \\
\hline Follow-up (months, median [IQR]) & $47.4[24.0]$ & $49.6[23.2]$ & $49.1[25.6]$ & $43.9[20.3]$ & 0.016 & $8.332^{\mathrm{a}}$ \\
\hline Composite endpoints $(n, \%)$ & $27(8.2)$ & $6(5.4)$ & $6(5.5)$ & $15(13.6)$ & 0.038 & $6.535^{\mathrm{b}}$ \\
\hline $\operatorname{ESRD}(n, \%)$ & $10(3.0)$ & $2(1.8)$ & $0(0.0)$ & $8(7.3)$ & 0.003 & $9.663^{\mathrm{b}}$ \\
\hline Use of RAAS blockade $(n, \%)$ & $151(45.8)$ & $48(43.2)$ & $57(52.3)$ & $46(41.8)$ & 0.241 & $2.847^{\mathrm{b}}$ \\
\hline Use of IS $(n, \%)$ & $236(71.5)$ & $76(68.5)$ & $80(73.4)$ & $80(72.7)$ & 0.679 & $0.774^{\mathrm{b}}$ \\
\hline
\end{tabular}

$P L R$ platelet-to-lymphocyte ratio, $I Q R$ interquartile range, $S D$ standard deviation, $M A P$ mean arterial pressure, $W B C$ white blood cell co nt, $S c r$ serum creatinine, $U A$ uric acid, $e G F R$ estimated glomerular filtration rate, $R A A S$ rein-angiotensin-aldosterone-system, $I S$ immune suppression. Composite endpoint was eGFR decrease by $50 \%$ or ESRD. ESRD was defined as eGFR $<15 \mathrm{~mL} / \mathrm{min} / 1.73 \mathrm{~m}^{2}$, initiation of dialysis or renal transplantation

${ }^{a}$ Test statistic value for Kruskal-Wallis test (for non-normally distributed continuous data)

${ }^{\mathrm{b}}$ Chi-square value for standard chi-squared test or Fisher's exact test (for categorical data)

${ }^{\mathrm{c}} F$-ratio value for one-way ANOVA (normally distributed continuous data)
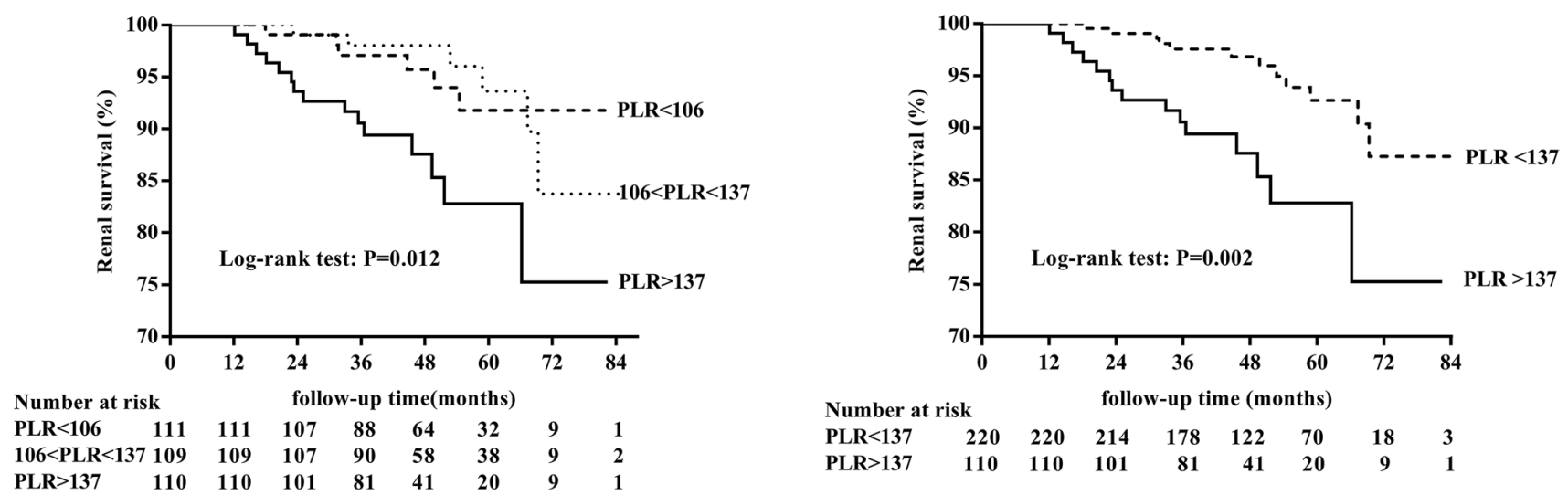

Fig. 2 Renal survival curves for the patients with IgAN according to the platelet-to-lymphocyte ratio

\section{Discussion}

Although the platelet count and lymphocyte count are routinely evaluated under simple laboratory conditions among the patients with IgAN, the clinical significance of PLR in the IgAN process remains unclear. Our study evaluated the relationship between PLR and the renal regression of IgAN and demonstrated that PLR $>137$ were independent 
Table 2 Univariate Cox regression analyses of renal survival in patients with IgAN

\begin{tabular}{lllr}
\hline Risk factor & \multicolumn{3}{l}{ Univariate } \\
\cline { 2 - 4 } & HR & $95 \%$ CI & \multicolumn{1}{r}{$P$-value } \\
\hline Age, years & 1.00 & $0.96-1.04$ & 0.962 \\
Male & 1.41 & $0.66-3.00$ & 0.376 \\
MAP, mmHg & 1.02 & $0.99-1.05$ & 0.128 \\
WBC, $\times 10^{9} / \mathrm{L}$ & 0.98 & $0.83-1.16$ & 0.806 \\
Lymphocytes, $\times 10^{9} / \mathrm{L}$ & 0.57 & $0.28-1.19$ & 0.134 \\
Platelets, $\times 10^{9} / \mathrm{L}$ & 1.00 & $0.99-1.01$ & 0.471 \\
PLR $>137$ & 3.10 & $1.44-6.64$ & $\mathbf{0 . 0 0 4}$ \\
Hemoglobin, g/L & 0.97 & $0.95-0.99$ & $\mathbf{0 . 0 0 1}$ \\
Serum albumin, g/l & 0.92 & $0.88-0.97$ & $\mathbf{0 . 0 0 1}$ \\
Scr, $\mu$ mol/L & 1.04 & $1.03-1.05$ & $<\mathbf{0 . 0 0 1}$ \\
UA, $\mu$ mol/L & 1.01 & $1.00-1.01$ & $<\mathbf{0 . 0 0 1}$ \\
eGFR, ml/min $/ 1.73 \mathrm{~m}^{2}$ & 0.92 & $0.90-0.95$ & $<\mathbf{0 . 0 0 1}$ \\
Proteinuria, g/24 h & 1.38 & $1.16-1.63$ & $<\mathbf{0 . 0 0 1}$ \\
Use of RAAS blockade & 0.59 & $0.27-1.30$ & 0.190 \\
Use of IS & 0.23 & $0.11-0.51$ & $<\mathbf{0 . 0 0 1}$ \\
\hline
\end{tabular}

IgAN Immunoglobulin A nephropathy, HRs hazard ratios, CIs confidence intervals, $P L R$ platelet-to-lymphocyte ratio, MAP mean arterial pressure, $W B C$ white blood cell count, $S c r$ serum creatinine, $U A$ uric acid, $e G F R$ estimated glomerular filtration rate, RAAS rein-angiotensin-aldosterone-system, $I S$ immune suppression

Table 3 Multivariate Cox regression analyses of renal survival in patients with IgAN

\begin{tabular}{llll}
\hline Risk factor & \multicolumn{3}{l}{ Multivariate } \\
\cline { 2 - 4 } & HR & $95 \%$ CI & $P$-value \\
\hline Age, years & 0.93 & $0.88-0.99$ & $\mathbf{0 . 0 1 9}$ \\
Male & 0.67 & $0.13-1.01$ & 0.637 \\
PLR $>137$ & 2.79 & $1.08-7.26$ & $\mathbf{0 . 0 3 5}$ \\
Hemoglobin, g/L & 0.98 & $0.96-1.01$ & 0.199 \\
Serum albumin, g/l & 0.97 & $0.89-1.07$ & 0.582 \\
Scr, $\mu$ mol/L & 1.00 & $0.95-1.05$ & 0.962 \\
UA, $\mu \mathrm{mol} / \mathrm{L}$ & 1.00 & $1.00-1.01$ & 0.925 \\
eGFR, ml/min/1.73 m ${ }^{2}$ & 0.94 & $0.87-1.02$ & 0.131 \\
Proteinuria, g/24 h & 1.20 & $0.86-1.68$ & 0.284 \\
Use of IS & 0.17 & $0.06-0.52$ & $\mathbf{0 . 0 0 2}$ \\
\hline
\end{tabular}

IgAN Immunoglobulin A nephropathy, HRs hazard ratios, CIs confidence intervals, $P L R$ platelet-to-lymphocyte ratio, $S c r$ serum creatinine, $U A$ uric acid, $e G F R$ estimated glomerular filtration rate, $I S$ immune suppression

prognostic factors for the long-term renal survival of patients with IgAN.

The advantage of the PLR is that it reflects the condition of patients in both inflammation and thrombosis pathways. It may be more valuable than either platelet or lymphocyte count alone. In our study, the mean value and corresponding 95\% reference interval for the PLR in IgAN patients was
125.8 (63.3-227.9), which was close to the PLR value in the general population reported in a population-based prospective cohort study: 120 (61-239) [23]. Most studies showed that elevated PLR value played a predictive role in several diseases [12, 24-27]. Our study found the same outcomes in IgAN. As we all know, platelets and lymphocytes are derived from the same hematopoietic stem cells and the PLR should be kept constant for homeostasis [28, 29]. Higher PLR condition means relatively high platelets and low lymphocytes. Higher platelet count may reflect increased thrombocyte activation, which contributes to increased inflammation and thrombocytosis, and thereby result in adverse renal outcomes [30, 31]. Lymphocytopenia, which reveals depression of innate cellular immunity, may be induced by the systemic inflammatory response, and be responsible for an inadequate immunologic reaction and a weakened defense $[32,33]$. Considering platelets and lymphocytes together, an elevated PLR might predict the bad body condition and weakening inflammatory response in those IgAN patients with poorer renal outcomes.

After Stratified analysis, we also found that the prognostic value of PLR remained the same for female patients or patients with eGFR less than $60 \mathrm{~mL} / \mathrm{min} / 1.73 \mathrm{~m}^{2}$. As we all know, females tend to mount stronger inflammatory responses than males [34-38], so the inflammation indicator, PLR, was more sensitive in female patients with IgAN. And for those IgAN patients with eGFR less than $60 \mathrm{~mL} / \mathrm{min} / 1.73 \mathrm{~m}^{2}$, they may have increased serum levels of inflammatory mediators and were more susceptible to inflammation-related vascular dysfunction and cardiovascular risk compared with healthy controls $[39,40]$. Turkmen et al. [24] carried out a cross-sectional study involving 62 ESRD patients prompted that a simple calculation of PLR can predict inflammation in ESRD patients. These may suggest that those IgAN patients with worse renal function have been in a long-term inflammatory state, so the inflammation factors occupy a larger proportion in the progression to renal failure.

Besides the PLR, hemoglobin, serum albumin, Scr, UA, eGFR, proteinuria, and use of IS treatments were also factors significantly associated with renal survival in the present study. Several clinical indicators such as renal function and proteinuria at biopsy are demonstrated to be associated with the renal outcome of IgAN [7-9, 41, 42]. Our study also found that the use of IS therapy was associated with better renal outcomes among IgAN patients. IS therapy was a common treatment for immune-mediated kidney disease, however, the role of the use of IS therapy in IgAN was still controversial [43-47]. Larger prospective studies with more patients and longer follow-up time were needed to assess the effect of IS therapy in IgAN.

This study had several limitations. The potential limitation of the present study was that it was a retrospective, 
Fig. 3 Subgroup analyses about the association of platelet-tolymphocyte ratio and renal survival according to sex, baseline eGFR, and baseline proteinuria for the patients with IgAN

Subgroup
All patients
Sex
male
female
eGFR
$\geq 60 \mathrm{ml} / \mathrm{min} / 1.73 \mathrm{~m}^{2}$
$<60 \mathrm{ml} / \mathrm{min} / 1.73 \mathrm{~m}^{2}$
Proteinuria
$<1 \mathrm{~g} / 24 \mathrm{~h}$
$\geq 1 \mathrm{~g} / 24 \mathrm{~h}$
330

Endpoint

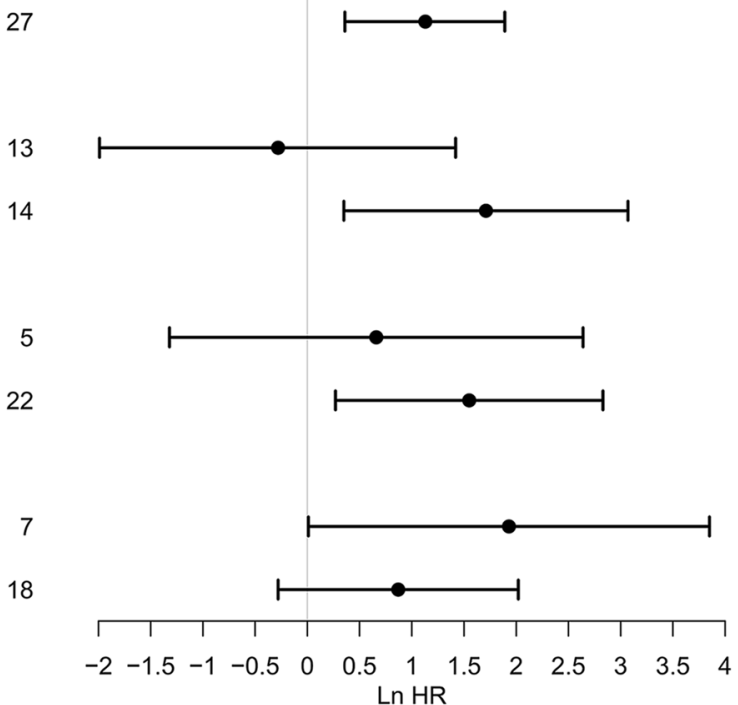

Ethical approval This is an observational study approved by the Medical Ethics Committee of the Tongji Hospital Affiliated with Tongji Medical College, Huazhong University of Science and Technology (approval number TJ-IRB20181108). All study procedures complied with the Declaration of Helsinki. All patients provided written informed consent to participate in the study.

Informed consent This study was performed after obtaining written informed consent from all patients.

Open Access This article is licensed under a Creative Commons Attribution 4.0 International License, which permits use, sharing, adaptation, distribution and reproduction in any medium or format, as long as you give appropriate credit to the original author(s) and the source, provide a link to the Creative Commons licence, and indicate if changes were made. The images or other third party material in this article are included in the article's Creative Commons licence, unless indicated otherwise in a credit line to the material. If material is not included in the article's Creative Commons licence and your intended use is not permitted by statutory regulation or exceeds the permitted use, you will need to obtain permission directly from the copyright holder. To view a copy of this licence, visit http://creativecommons.org/licenses/by/4.0/.

\section{References}

Funding This work was financially supported by Major Research Plan of the National Natural Science Foundation of China (Grant No. 91742204, 81470948, 81670633, 81570667), International (regional) cooperation and exchange projects, (NSFC-DFG, Grant No. 81761138041), National key research and development program (Grants 2016YFC0906103, 2013BAI09B06, 2015BAI12B07).

\section{Compliance with ethical standards}

Conflict of interest Authors of this study have no conflict of interest.

1. Wyatt RJ, Julian BA (2013) IgA nephropathy. N Engl J Med 368(25):2402-2414

2. Yeo SC, Goh SM, Barratt J (2019) Is immunoglobulin A nephropathy different in different ethnic populations? Nephrology (Carlton) 24(9):885-895

3. Barbour SJ, Cattran DC, Kim SJ et al (2013) Individuals of Pacific Asian origin with IgA nephropathy have an increased risk of progression to end-stage renal disease. Kidney Int 84(5):1017-1024 
4. Li LS, Liu ZH (2004) Epidemiologic data of renal diseases from a single unit in China: analysis based on 13,519 renal biopsies. Kidney Int 66(3):920-923

5. Bikbov B, Purcell CA, Levey AS et al (2020) Global, regional, and national burden of chronic kidney disease, 1990-2017: a systematic analysis for the Global Burden of Disease Study 2017. Lancet 395(10225):709-733

6. Schena FP, Cox SN (2018) Biomarkers and precision medicine in IgA nephropathy. Semin Nephrol 38(5):521-530

7. Barbour SJ, Reich HN (2012) Risk stratification of patients with IgA nephropathy. Am J Kidney Dis 59(6):865-873

8. Barbour S, Reich H (2018) An update on predicting renal progression in IgA nephropathy. Curr Opin Nephrol Hypertens 27(3):214-220

9. Le W, Liang S, Hu Y et al (2012) Long-term renal survival and related risk factors in patients with $\mathrm{IgA}$ nephropathy: results from a cohort of 1155 cases in a Chinese adult population. Nephrol Dial Transplant 27(4):1479-1485

10. Templeton AJ, Ace O, McNamara MG et al (2014) Prognostic role of platelet to lymphocyte ratio in solid tumors: a systematic review and meta-analysis. Cancer Epidemiol Biomark Prevent 23(7):1204-1212

11. Li DY, Hao XY, Ma TM, Dai HX, Song YS (2017) The prognostic value of platelet-to-lymphocyte ratio in urological cancers: a meta-analysis. Sci Rep 7(1):15387

12. Wang J, Liu Y, Zhang N et al (2017) Prognostic role of pretreatment platelet to lymphocyte ratio in urologic cancer. Oncotarget 8(41):70874-70882

13. Wang Z, Peng S, Wang A et al (2018) Platelet-lymphocyte ratio acts as an independent predictor of prognosis in patients with renal cell carcinoma. Clin Chim Acta 480:166-172

14. Sun XP, Li J, Zhu WW et al (2017) Impact of platelet-to-lymphocyte ratio on clinical outcomes in patients with ST-segment elevation myocardial infarction. Angiology 68(4):346-353

15. Lee YSG, Baradi A, Peverelle M et al (2018) Usefulness of platelet-to-lymphocyte ratio to predict long-term all-cause mortality in patients at high risk of coronary artery disease who underwent coronary angiography. Am J Cardiol 121(9):1021-1026

16. Yao C, Liu X, Tang Z (2017) Prognostic role of neutrophillymphocyte ratio and platelet-lymphocyte ratio for hospital mortality in patients with AECOPD. Int J Chronic Obstr Pulm Dis 12:2285-2290

17. Cerit L (2017) Platelet to lymphocyte ratio and saphenous vein graft disease. Angiology 68(3):29

18. Rollino C, Vischini G, Coppo R (2016) IgA nephropathy and infections. J Nephrol 29(4):463-468

19. Sakai H (1988) Cellular immunoregulatory aspects of $\operatorname{IgA}$ nephropathy. Am J Kidney Dis 12(5):430-432

20. Rauen T, Floege J (2017) Inflammation in IgA nephropathy. Pediatr Nephrol 32(12):2215-2224

21. Wang J, Anders RA, Wu Q et al (2004) Dysregulated LIGHT expression on $\mathrm{T}$ cells mediates intestinal inflammation and contributes to IgA nephropathy. J Clin Invest 113(6):826-835

22. Levey AS, Bosch JP, Lewis JB et al (1999) A more accurate method to estimate glomerular filtration rate from serum creatinine: a new prediction equation. Modification of Diet in Renal Disease Study Group. Ann Intern Med 130(6):461-470

23. Fest J, Ruiter R, Ikram MA et al (2018) Reference values for white blood-cell-based inflammatory markers in the Rotterdam Study: a population-based prospective cohort study. Sci Rep 8(1):10566

24. Turkmen K, Erdur FM, Ozcicek F et al (2013) Platelet-to-lymphocyte ratio better predicts inflammation than neutrophil-tolymphocyte ratio in end-stage renal disease patients. Hemodial Int 17(3):391-396
25. Cao W, Yao X, Cen D et al (2020) The prognostic role of plateletto-lymphocyte ratio on overall survival in gastric cancer: a systematic review and meta-analysis. BMC Gastroenterol 20(1):16

26. Bao Y, Wang Y, Li X et al (2019) Prognostic significance of platelet-to-lymphocyte ratio in urothelial carcinoma patients: a meta-analysis. Cancer Cell Int 19:315

27. Tsai SF, Wu MJ, Wen MC, Chen CH (2019) Serologic and histologic predictors of long-term renal outcome in biopsy-confirmed IgA nephropathy (Haas Classification): an observational study. J Clin Med 8(6):848

28. Dharampuriya PR, Scapin G, Wong C et al (2017) Tracking the origin, development, and differentiation of hematopoietic stem cells. Curr Opin Cell Biol 49:108-115

29. Orkin SH, Zon LI (2008) Hematopoiesis: an evolving paradigm for stem cell biology. Cell 132(4):631-644

30. Akkaya E, Gul M, Ugur M (2014) Platelet to lymphocyte ratio: a simple and valuable prognostic marker for acute coronary syndrome. Int J Cardiol 177(2):597-598

31. Balta S, Demirkol S, Kucuk U (2013) The platelet lymphocyte ratio may be useful inflammatory indicator in clinical practice. Hemodial Int 17(4):668-669

32. Egido J, Blasco R, Sancho J, Lozano L (1983) T-cell dysfunctions in IgA nephropathy: specific abnormalities in the regulation of IgA synthesis. Clin Immunol Immunopathol 26(2):201-212

33. Menges T, Engel J, Welters I et al (1999) Changes in blood lymphocyte populations after multiple trauma: association with posttraumatic complications. Crit Care Med 27(4):733-740

34. Klein SL, Flanagan KL (2016) Sex differences in immune responses. Nat Rev Immunol 16(10):626-638

35. Wu H, Lai CF, Chang-Panesso M, Humphreys BD (2020) Proximal tubule translational profiling during kidney fibrosis reveals proinflammatory and long noncoding RNA expression patterns with sexual dimorphism. J Am Soc Nephrol 31(1):23-38

36. Dolsen MR, Crosswell AD, Prather AA (2019) Links between stress, sleep, and inflammation: are there sex differences? Curr Psychiatry Rep 21(2):8

37. Slominski B, Skrzypkowska M, Ryba-Stanislawowska M, Brandt A (2018) Sex-related association of serum uric acid with inflammation, kidney function and blood pressure in type 1 diabetic patients. Pediatr Diabetes 19(5):1014-1019

38. Ichii O, Nakamura T, Irie $T$ et al (2018) Close pathological correlations between chronic kidney disease and reproductive organassociated abnormalities in female cotton rats. Exp Biol Med (Maywood) 243(5):418-427

39. Dogra G, Irish A, Chan D, Watts G (2006) Insulin resistance, inflammation, and blood pressure determine vascular dysfunction in CKD. Am J Kidney Dis 48(6):926-934

40. Bernelot Moens SJ, Verweij SL, van der Valk FM et al (2017) Arterial and cellular inflammation in patients with CKD. J Am Soc Nephrol 28(4):1278-1285

41. Xie J, Lv J, Wang W et al (2018) Kidney failure risk prediction equations in IgA nephropathy: a multicenter risk assessment study in Chinese patients. Am J Kidney Dis 72(3):371-380

42. Zhu B, Liu WH, Yu DR et al (2020) The association of low hemoglobin levels with IgA nephropathy progression: a two-center cohort study of 1,828 Cases. Am J Nephrol 51(8):624-634

43. Chen T, Xia E, Chen $\mathrm{T}$ et al (2020) Identification and external validation of $\operatorname{IgA}$ nephropathy patients benefiting from immunosuppression therapy. EBioMedicine 52:102657

44. Rauen T, Fitzner C, Eitner F et al (2018) Effects of two immunosuppressive treatment protocols for IgA nephropathy. J Am Soc Nephrol 29(1):317-325

45. Jefferson JA (2018) Complications of immunosuppression in glomerular disease. Clin J Am Soc Nephrol 13(8):1264-1275

46. Floege J, Rauen T (2016) Immunosuppression in IgA nephropathy: how certain are we? Kidney Int 89(1):9-11 
47. Rauen T, Eitner F, Fitzner C et al (2015) Intensive supportive care plus immunosuppression in IgA nephropathy. N Engl J Med 373(23):2225-2236

48. Avramovski P, Avramovska M, Sotiroski K, Sikole A (2019) Acute-phase proteins as promoters of abdominal aortic calcification in chronic dialysis patients. Saudi J Kidney Dis Transplant 30(2):376-386
Publisher's Note Springer Nature remains neutral with regard to jurisdictional claims in published maps and institutional affiliations. 